\title{
Boundary Feedback Stabilization of a Vibrating String with an Interior Point Mass
}

\author{
W. Littman \\ School of Mathematics \\ University of Minnesota \\ Minneapolis \\ MN 55455, USA
}

\author{
S. W. Taylor \\ Department of Mathematics \\ University of Auckland \\ Private Bag 92019 \\ Auckland, New Zealand
}

\begin{abstract}
We study the boundary feedback stabilization for a one-dimensional wave equation with an interior point mass. We show that if the initial data belong to a certain invariant subspace of the semigroup of operators that generates the solution of the system, then the energy will decay like $C$ /time. This improves a result of Hansen and Zuazua [1] who consider decay of solutions belonging to the domain of a power of the infinitesimal generator of the semigroup.
\end{abstract}

\section{Introduction.}

In this paper we study the boundary feedback stabilization for a one-dimensional wave equation with an interior point mass. We show that if the initial data belong to a certain invariant subspace of the semigroup of operators that generates the solution of the system, then the energy will decay like $C$ /time. This improves a result of Hansen and Zuazua [1] who consider decay of solutions belonging to the domain of a power of the infinitesimal generator of the semigroup. The system under investigation consists of two strings of length $l_{1}$ and $l_{2}$ respectively. In their rest states, the strings occupy the intervals $\Omega_{1}=\left(-l_{1}, 0\right)$ and $\Omega_{2}=\left(0, l_{2}\right)$ of the $x$-axis respectively. At the origin, each string is tied to a particle of mass $M$ whose displacement away from the $x$-axis at time $t$ is given by $z(t)$. The transverse displacements of the strings are given by $u$ and $v$. In this model, the densities $\rho_{1}, \rho_{2}$ and the tensions $\sigma_{1}, \sigma_{2}$ are assumed constant.

The equations satisfied by the system are listed below (more details are given in [1].)

$$
\left.\begin{array}{ll}
\rho_{1} u_{t t}=\sigma_{1} u_{x x}, & x \in \Omega_{1}, t>0, \\
\rho_{2} v_{t t}=\sigma_{2} v_{x x}, & x \in \Omega_{2}, t>0, \\
M z_{t t}+\sigma_{1} u_{x}(0, t)-\sigma_{2} v_{x}(0, t)=0, & t>0, \\
u(x, 0)=u^{0}(x), u_{t}(x, 0)=u^{1}(x), & x \in \Omega_{1}, \\
v(x, 0)=v^{0}(x), v_{t}(x, 0)=v^{1}(x), & x \in \Omega_{2} .
\end{array}\right\}
$$


We are interested in the problem of velocity feedback at one end. Note that Hansen and Zuazua [1] consider also the case of velocity feedback at both ends, and they show that energy decays uniformly exponentially. We assume that the velocity feedback occurs at the end $x=l_{2}$, while at the other end $x=-l_{1}$ we simply have a Dirichlet boundary condition. The boundary conditions are thus

$$
\left.\begin{array}{ll}
u\left(-l_{1}, t\right)=0, & t>0 \\
\sigma_{2} v_{x}\left(l_{2}, t\right)+\gamma v_{t}\left(l_{2}, t\right)=0, & t>0
\end{array}\right\}
$$

where $\gamma$ is positive.

\section{A Representation of the Solution.}

We can simplify the exposition by scaling the space variable $x$ separately for $x<0$ and for $x>0$ so that the wave speed of each wave equation becomes unity. This is achieved by considering a new variable

$$
\tilde{x}= \begin{cases}x\left(\rho_{1} / \sigma_{1}\right)^{1 / 2}, & x<0 \\ x\left(\rho_{2} / \sigma_{2}\right)^{1 / 2}, & x \geq 0 .\end{cases}
$$

Thus, we may consider without loss of generality the following system (tildes have been removed)

$$
\left.\begin{array}{ll}
u_{t t}=u_{x x}, & x \in \Omega_{1}, t>0, \\
v_{t t}=v_{x x}, & x \in \Omega_{2}, t>0, \\
M z_{t t}+\mu_{1} u_{x}(0, t)-\mu_{2} v_{x}(0, t)=0, & t>0, \\
u(x, 0)=u^{0}(x), u_{t}(x, 0)=u^{1}(x), & x \in \Omega_{1}, \\
v(x, 0)=v^{0}(x), v_{t}(x, 0)=v^{1}(x), & x \in \Omega_{1},
\end{array}\right\}
$$

where the modified tensions are given by $\mu_{1}=\left(\rho_{1} \sigma_{1}\right)^{1 / 2}, \mu_{2}=\left(\rho_{2} \sigma_{2}\right)^{1 / 2}$. The boundary conditions now become

$$
\left.\begin{array}{ll}
u\left(-l_{1}, t\right)=0, & t>0 \\
\mu_{2} v_{x}\left(l_{2}, t\right)+\gamma v_{t}\left(l_{2}, t\right)=0, & t>0
\end{array}\right\}
$$

and the total mechanical energy simplifies to

$$
\begin{aligned}
\mathcal{E}(t) & =\frac{M}{2}\left|z_{t}(t)\right|^{2}+\frac{\mu_{1}}{2} \int_{-l_{1}}^{0}\left|u_{t}(x, t)\right|^{2}+\left|u_{x}(x, t)\right|^{2} d x \\
& +\frac{\mu_{2}}{2} \int_{0}^{l_{2}}\left|v_{t}(x, t)\right|^{2}+\left|v_{x}(x, t)\right|^{2} d x .
\end{aligned}
$$

We define the finite energy space

$$
\mathcal{H}=\left\{\left(\begin{array}{c}
U^{0} \in H^{1}\left(\Omega_{1}\right), \\
V^{0} \in H^{1}\left(\Omega_{2}\right), \\
Z^{0} \in \mathbb{R}, \\
U^{1} \in L^{2}\left(\Omega_{1}\right), \\
V^{1} \in L^{2}\left(\Omega_{2}\right), \\
Z^{1} \in \mathbb{R}
\end{array}\right): \begin{array}{c}
U^{0}\left(-l_{1}\right)=0, \\
U^{0}(0)=V^{0}(0)=Z^{0}
\end{array}\right\}
$$


and equip $\mathcal{H}$ with the norm

$$
\begin{aligned}
\left\|\left(U^{0}, V^{0}, Z^{0}, U^{1}, V^{1}, Z^{1}\right)\right\| & =\left(\mu_{1} \int_{-l_{1}}^{0}\left|U^{1}(x)\right|^{2}+\left|U_{x}^{0}(x)\right|^{2} d x\right. \\
& \left.+M\left|Z^{1}\right|^{2}+\mu_{2} \int_{0}^{l_{2}}\left|V^{1}(x)\right|^{2}+\left|V_{x}^{0}(x)\right|^{2} d x\right)^{1 / 2} .
\end{aligned}
$$

It is easy to see that $\mathcal{H}$ is a Hilbert space, and we define on $\mathcal{H}$ the operator $\mathcal{A}$, with domain $D(\mathcal{A})=\left\{\left(U^{0}, V^{0}, Z^{0}, U^{1}, V^{1}, Z^{1}\right) \in \mathcal{H} \cap\left(H^{2}\left(\Omega_{1}\right) \times H^{2}\left(\Omega_{2}\right) \times \mathbb{R} \times H^{1}\left(\Omega_{1}\right) \times H^{1}\left(\Omega_{2}\right) \times \mathbb{R}\right):\right.$ $\left.U^{1}\left(-l_{1}\right)=0, U^{1}(0)=V^{1}(0)=Z^{1}, \mu_{2} V_{x}^{0}\left(l_{2}\right)+\gamma V^{1}\left(l_{2}\right)=0\right\}$, given by

$$
\mathcal{A}\left(U^{0}, V^{0}, Z^{0}, U^{1}, V^{1}, Z^{1}\right)=\left(U^{1}, V^{1}, Z^{1}, U_{x x}^{0}, V_{x x}^{0},\left(\mu_{2} V_{x}^{0}(0)-\mu_{1} U_{x}(0)\right) / M\right) .
$$

As is mentioned in [1], it is easy to check that $\mathcal{A}$ is the infinitesimal generator of a strongly continuous semigroup of contractions $T(t)$ on $\mathcal{H}$ (the Lumer Phillips Theorem, which is stated in [6], can be used to deduce this). The finite energy solutions of (3), (4) are then given by

$$
\left(u(., t), v(., t), z(t), u_{t}(., t), v_{t}(., t), z_{t}(t)\right)=T(t)\left(u^{0}, v^{0}, z^{0}, u^{1}, v^{1}, z^{1}\right) .
$$

A convenient way to analyze the decay of solutions of (3), (4) is to use the fact that the solution of each of the wave equations is a sum of two waves, one moving to the left and the other to the right:

$$
\left.\begin{array}{ll}
u(x, t)=F\left(x+t+l_{1}\right)-G(t-x), & x \in \Omega_{1}, t \geq 0 \\
v(x, t)=H(x+t)-E\left(t-x+l_{2}\right), & x \in \Omega_{2}, t \geq 0 .
\end{array}\right\}
$$

The regularity of the functions $F, G, H$ and $E$ is easily checked, because we have $F^{\prime}(x+$ $\left.t+L_{1}\right)=\frac{1}{2}\left(u_{x}(x, t)+u_{t}(x, t)\right), G^{\prime}\left(x+t+L_{1}\right)=\frac{1}{2}\left(u_{x}(x, t)-u_{t}(x, t)\right)$, etc. and thus for finite energy solutions, the functions $F, G, H$ and $E$ are locally in $H^{1}$. Moreover, because of the strong continuity of the semigroup, it follows that $z(t)=F\left(t+l_{1}\right)-G(t)=H(t)-E\left(t+l_{2}\right)$ is $C^{1}$. If the initial data happens to be in the domain of $\mathcal{A}$, then it is easily seen that all of these functions have one more derivative, i.e. $F, G, H$ and $E$ are locally in $H^{2}$ and $z(t)=F\left(t+l_{1}\right)-G(t)=H(t)-E\left(t+l_{2}\right)$ is $C^{2}$.

In terms of these functions, the energy (5) may be written

$$
\begin{aligned}
\mathcal{E}(t) & =\mu_{1} \int_{-l_{1}}^{0}\left|F^{\prime}(t-x)\right|^{2}+\left|G^{\prime}(t-x)\right|^{2} d x \\
& +\mu_{2} \int_{0}^{l_{2}}\left|E^{\prime}(t+x)\right|^{2}+\left|H^{\prime}(t+x)\right|^{2} d x \\
& +\frac{1}{2} M\left|\frac{d}{d t}\left(F\left(t+l_{1}\right)-G(t)\right)\right|^{2} .
\end{aligned}
$$

The initial conditions of (3) imply that the functions $F, G, H$ and $E$ satisfy, modulo some irrelevant arbitrary constants,

$$
\left.\begin{array}{ll}
F(s)=\frac{1}{2} u^{0}\left(s-l_{1}\right)+\frac{1}{2} \int_{0}^{s-l_{1}} u^{1}(\sigma) d \sigma, & 0<s<l_{1}, \\
G(s)=-\frac{1}{2} u^{0}(-s)+\frac{1}{2} \int_{0}^{-s} u^{1}(\sigma) d \sigma, & 0<s<l_{1}, \\
H(s)=\frac{1}{2} v^{0}(s)+\frac{1}{2} \int_{0}^{s} v^{1}(\sigma) d \sigma, & 0<s<l_{2}, \\
E(s)=-\frac{1}{2} v^{0}\left(l_{2}-s\right)+\frac{1}{2} \int_{0}^{l_{2}-s} v^{1}(\sigma) d \sigma, & 0<s<l_{2} .
\end{array}\right\}
$$


The values of these functions for other positive values of their arguments are found by solving a system of differential-delay equations, which are obtained from the conditions at $x=0$ of (3) and the boundary conditions (4). Specifically, for $s>0$,

$$
\left.\begin{array}{ll}
G\left(s+l_{1}\right) & =F(s), \\
F\left(s+l_{1}\right)+E\left(s+l_{2}\right) & =G(s)+H(s), \\
H^{\prime}\left(s+l_{2}\right) & =q E^{\prime}(s), \\
M\left(F\left(s+l_{1}\right)-G(s)\right)_{s s} & =\mu_{2}\left(H^{\prime}(s)+E^{\prime}\left(s+l_{2}\right)\right) \\
& -\mu_{1}\left(F^{\prime}\left(s+l_{1}\right)+G^{\prime}(s)\right),
\end{array}\right\}
$$

where $q=\left(\gamma-\mu_{2}\right) /\left(\gamma+\mu_{2}\right)$. There are two points that should be mentioned before we proceed. The first is that $|q|<1$ which will be very important in the sequel. The second is that Equations (9) require more smoothness than finite energy solutions possess. However, we use (9) only to construct explicit formulae for the Laplace transforms (see (10) below) of $F^{\prime}, G^{\prime}, H^{\prime}$ and $E^{\prime}$. But because (9) are valid for initial data that are in the domain of $\mathcal{A}$, a standard density argument may be used to show that the formulae for our Laplace transforms are valid for all finite energy solutions. Since the argument is straightforward, we shall omit any further details of it.

We define

$$
\begin{aligned}
& f(\lambda)=\int_{0}^{\infty} e^{-\lambda t} F^{\prime}(t) d t, \quad f^{1}(\lambda)=\int_{0}^{l_{1}} e^{-\lambda t} F^{\prime}(t) d t \\
& g(\lambda)=\int_{0}^{\infty} e^{-\lambda t} G^{\prime}(t) d t, \quad g^{1}(\lambda)=\int_{0}^{l_{1}} e^{-\lambda t} G^{\prime}(t) d t, \\
& h(\lambda)=\int_{0}^{\infty} e^{-\lambda t} H^{\prime}(t) d t, \quad h^{1}(\lambda)=\int_{0}^{l_{2}} e^{-\lambda t} H^{\prime}(t) d t, \\
& e(\lambda)=\int_{0}^{\infty} e^{-\lambda t} E^{\prime}(t) d t, \quad e^{1}(\lambda)=\int_{0}^{l_{2}} e^{-\lambda t} E^{\prime}(t) d t . \quad
\end{aligned}
$$

Note that the Laplace transforms in Equations (10) all exist for $\operatorname{Re} \lambda>0$. This is because for finite energy solutions, $F^{\prime}, G^{\prime}, H^{\prime}$ and $E^{\prime}$ have $L^{2}$ norms on any bounded subinterval of $(0, \infty)$ that are bounded by constants that depend on the length, but not the location, of the subinterval. The inversion of these transforms will be important to us. One could use an inverse Laplace transform approach, but we prefer to extend the functions $F^{\prime}, G^{\prime}, H^{\prime}$ and $E^{\prime}$ to be zero on $(-\infty, 0)$ and interpret these transforms as Fourier transforms. Thus, if $\lambda=\sigma+i \xi$ and $\sigma>0$ then $f(\lambda)$ is just the Fourier transform of $e^{-\sigma t} F^{\prime}(t)$.

Formally, Equations (9) imply that

$$
\begin{aligned}
0 & =g(\lambda)-g^{1}(\lambda)-f(\lambda) e^{-l_{1} \lambda} \\
0 & =e^{l_{1} \lambda}\left(f(\lambda)-f^{1}(\lambda)\right)-g(\lambda)-h(\lambda)+e^{l_{2} \lambda}\left(e(\lambda)-e^{1}(\lambda)\right) \\
0 & =h(\lambda)-h^{1}(\lambda)-e^{-l_{2} \lambda} q e(\lambda) \\
0 & =M\left(\lambda e^{l_{1} \lambda}\left(f(\lambda)-f^{1}(\lambda)\right)-F^{\prime}\left(l_{1}\right)+G^{\prime}(0)-\lambda g(\lambda)\right) \\
& -\mu_{2}\left(h(\lambda)+e^{l_{2} \lambda}\left(e(\lambda)-e^{1}(\lambda)\right)\right. \\
& +\mu_{1}\left(e^{l_{1} \lambda}\left(f(\lambda)-f^{1}(\lambda)\right)+g(\lambda)\right)
\end{aligned}
$$

The solutions of these equations are easily found. First we define

$$
\begin{aligned}
Q(\lambda)= & \frac{1+q e^{-2 l_{2} \lambda}}{1-q e^{-2 l_{2} \lambda}}, \\
S(\lambda)= & \left(1-q e^{-2 l_{2} \lambda}\right)^{-1}\left(\left[M \lambda+\mu_{1}+Q(\lambda) \mu_{2}\right]\right. \\
& \left.-e^{-2 l_{1} \lambda}\left[M \lambda-\mu_{1}+Q(\lambda) \mu_{2}\right]\right)^{-1},
\end{aligned}
$$


and now we may write the solutions of System (11) as follows:

$$
\begin{aligned}
f(\lambda) & =\left\{f^{1}(\lambda)\left[M \lambda+\mu_{1}+\mu_{2}-q e^{-2 l_{2} \lambda}\left(M \lambda+\mu_{1}-\mu_{2}\right)\right]\right. \\
& +g^{1}(\lambda) e^{-l_{1} \lambda}\left[M \lambda-\mu_{1}+\mu_{2}-q e^{-2 l_{2} \lambda}\left(M \lambda-\mu_{1}-\mu_{2}\right)\right] \\
& +2 \mu_{2} h^{1}(\lambda) e^{-l_{1} \lambda}+2 q \mu_{2} e^{1}(\lambda) e^{-\left(l_{1}+l_{2}\right) \lambda} \\
& \left.+M\left(F^{\prime}\left(l_{1}\right)-G^{\prime}(0)\right) e^{-l_{1} \lambda}\left(1-q e^{-2 l_{2} \lambda}\right)\right\} S(\lambda), \\
g(\lambda) & =\left\{g^{1}(\lambda)\left[M \lambda+\mu_{1}+\mu_{2}-q e^{-2 l_{2} \lambda}\left(M \lambda+\mu_{1}-\mu_{2}\right)\right]\right. \\
& +f^{1}(\lambda) e^{-l_{1} \lambda}\left[M \lambda+\mu_{1}+\mu_{2}-q e^{-2 l_{2} \lambda}\left(M \lambda+\mu_{1}-\mu_{2}\right)\right] \\
& +2 \mu_{2} h^{1}(\lambda) e^{-2 l_{1} \lambda}+2 q \mu_{2} e^{1}(\lambda) e^{-2 l_{1} \lambda} \\
& \left.+M\left(F^{\prime}\left(l_{1}\right)-G^{\prime}(0)\right) e^{-2 l_{1} \lambda}\left(1-q e^{-2 l_{2} \lambda}\right)\right\} S(\lambda), \\
& \\
h(\lambda) & =\left\{h^{1}(\lambda)\left[M \lambda+\mu_{1}+\mu_{2}-e^{-2 l_{1} \lambda}\left(M \lambda-\mu_{1}+\mu_{2}\right)\right]\right. \\
& +e^{1}(\lambda) q e^{-l_{2} \lambda}\left[M \lambda+\mu_{1}+\mu_{2}-e^{-2 l_{1} \lambda}\left(M \lambda-\mu_{1}+\mu_{2}\right)\right] \\
& +2 \mu_{1} q f^{1}(\lambda) e^{-\left(l_{1}+2 l_{2}\right) \lambda}+2 q \mu_{1} g^{1}(\lambda) e^{-2 l_{2} \lambda} \\
& \left.-q M\left(F^{\prime}\left(l_{1}\right)-G^{\prime}(0)\right) e^{-2 l_{2} \lambda}\left(1-e^{-2 l_{1} \lambda}\right)\right\} S(\lambda), \\
& \\
e(\lambda) & =\left\{e^{1}(\lambda)\left[M \lambda+\mu_{1}+\mu_{2}-e^{-2 l_{1} \lambda}\left(M \lambda-\mu_{1}+\mu_{2}\right)\right]\right. \\
& +h^{1}(\lambda) e^{-l_{2} \lambda}\left[M \lambda+\mu_{1}-\mu_{2}-e^{-2 l_{1} \lambda}\left(M \lambda-\mu_{1}-\mu_{2}\right)\right] \\
& +2 \mu_{1} q f^{1}(\lambda) e^{-\left(l_{1}+l_{2}\right) \lambda}+2 \mu_{1} g^{1}(\lambda) e^{-l_{2} \lambda} \\
& \left.-M\left(F^{\prime}\left(l_{1}\right)-G^{\prime}(0)\right) e^{-l_{2} \lambda}\left(1-e^{-2 l_{1} \lambda}\right)\right\} S(\lambda) .
\end{aligned}
$$

Lemma $1 Q(\lambda)$ and $S(\lambda)$ satisfy the following inequalities:

1.

$$
\frac{1-|q| e^{-2 l_{2} \operatorname{Re} \lambda}}{1+|q| e^{-2 l_{2} \operatorname{Re} \lambda}} \leq \operatorname{Re} Q(\lambda) \leq \frac{1+|q| e^{-2 l_{2} \operatorname{Re} \lambda}}{1-|q| e^{-2 l_{2} \operatorname{Re} \lambda}}
$$

2. There exists a constant $C$ such that if $\operatorname{Re} \lambda>0$ then

$$
|S(\lambda)|<\frac{C}{1+|\lambda|}\left(1-e^{-2 l_{1} \operatorname{Re} \lambda}\right)^{-1}
$$

Proof. A simple calculation shows that

$$
\operatorname{Re} Q(\sigma+i \xi)=\frac{1-q^{2} e^{-4 l_{2} \sigma}}{1-2 q e^{-2 l_{2} \sigma} \cos \xi+q^{2} e^{-4 l_{2} \sigma}} .
$$

Hence

$$
\frac{1-|q| e^{-2 l_{2} \sigma}}{1+|q| e^{-2 l_{2} \sigma}} \leq \operatorname{Re} Q(\sigma+i \xi) \leq \frac{1+|q| e^{-2 l_{2} \sigma}}{1-|q| e^{-2 l_{2} \sigma}}
$$

which proves the first inequality. But $|q|<1$, so $\operatorname{Re} Q(\sigma+i \xi)>0$ in the set $\sigma>\frac{\log |q|}{2 l_{2}}$, which contains the right half plane. Hence,

$$
\left|\operatorname{Re}\left(M \lambda+\mu_{1}+Q(\lambda) \mu_{2}\right)\right|>\left|\operatorname{Re}\left(M \lambda-\mu_{1}+Q(\lambda) \mu_{2}\right)\right|
$$


if $\operatorname{Re} \lambda \geq 0$. It is clear that

$$
\operatorname{Im}\left(M \lambda+\mu_{1}+Q(\lambda) \mu_{2}\right)=\operatorname{Im}\left(M \lambda-\mu_{1}+Q(\lambda) \mu_{2}\right),
$$

so

$$
\left|M \lambda+\mu_{1}+Q(\lambda) \mu_{2}\right|>\left|M \lambda-\mu_{1}+Q(\lambda) \mu_{2}\right|
$$

if $\operatorname{Re} \lambda \geq 0$, and thus

$$
|S(\lambda)| \leq\left|\left(1-q e^{-2 l_{2} \lambda}\right)^{-1}\left(M \lambda+\mu_{1}+Q(\lambda) \mu_{2}\right)^{-1}\right|\left(1-e^{-2 l_{1} \operatorname{Re} \lambda}\right)^{-1} .
$$

The second inequality in the statement of the lemma follows easily from this.

\section{Energy Decay Estimates.}

In this section we analyze the decay of energy of the string-mass system. We find that the energy of the string to the right of the particle (i.e. the part of the string corresponding to the interval $\left(0, l_{2}\right)$ of the $x$-axis) decays uniformly when the initial data have finite energy. A similar decay rate holds for the remainder of the energy, but this requires an extra derivative in the initial data for the part of the string to the left of the particle.

In order to prove our results, it is useful to consider for $m=1,2, \ldots$, the following approximations $S_{m}(\lambda)$ to $S(\lambda)$.

$$
S_{m}(\lambda)=\left(1-q e^{-2 l_{2} \lambda}\right)^{-1} \sum_{k=0}^{m} \frac{e^{-2 k l_{1} \lambda}\left[M \lambda-\mu_{1}+Q(\lambda) \mu_{2}\right]^{k}}{\left[M \lambda+\mu_{1}+Q(\lambda) \mu_{2}\right]^{k+1}} .
$$

Lemma $2 \lim _{m \rightarrow \infty} S_{m}(\lambda)=S(\lambda)$, with uniform convergence on sets of the form $\{\lambda: \operatorname{Re} \lambda \geq$ $\sigma\}$, where $\sigma>0$.

Proof. The stated convergence properties of the geometric series are an immediate consequence of Inequality (18).

We will be able to simplify our proofs of energy decay by considering only solutions of the string-mass systems for which the initial velocity of the point mass is zero. We will achieve this simplification by subtracting an eigenfunction solution from the solution of (3) and (4). The fact that this can be done is due to the fact that the eigenvector components corresponding to the velocity and displacement of the point mass cannot vanish. We prove this in the following lemma:

Lemma 3 If $\left(U^{0}, V^{0}, Z^{0}, U^{1}, V^{1}, Z^{1}\right)$ is an eigenvector of $\mathcal{A}$ then $Z^{0}$ and $Z^{1}$ are non-zero real numbers.

Proof. Hansen and Zuazua prove in [1] that the eigenvalues of $\mathcal{A}$ lie in the left half plane. If $\lambda$ is such an eigenvalue then $Z^{1}=\lambda Z^{0}$ and $U^{0}(0)=Z^{0}$. Thus if one of $Z^{0}$ or $Z^{1}$ vanished then they would both vanish and $U^{0}$ would satisfy

$$
U_{x x}^{0}=\lambda^{2} U^{0}, \quad U^{0}\left(-l_{1}\right)=U^{0}(0)=0 .
$$


This has only trivial solutions because $\lambda$ is in the left half plane. Hence $U^{0}=0$. Thus, $V^{0}$ must satisfy

$$
V_{x x}^{0}=\lambda^{2} V^{0}, \quad V_{x}^{0}(0)=V^{0}(0)=0,
$$

which implies that $V^{0}$ is also zero. It is now easy to see that all of the components of the eigenvector are zero, which is impossible.

We now consider the energy of different parts of the solution. Specifically, we write $\mathcal{E}=\mathcal{E}_{1}+\mathcal{E}_{2}+\mathcal{E}_{3}$, where

$$
\left.\begin{array}{rl}
\mathcal{E}_{1}(t) & =\frac{\mu_{1}}{2} \int_{-l_{1}}^{0}\left|u_{t}(x, t)\right|^{2}+\left|u_{x}(x, t)\right|^{2} d x \\
& =\mu_{1} \int_{-l_{1}}^{0}\left|F^{\prime}(t-x)\right|^{2}+\left|G^{\prime}(t-x)\right|^{2} d x, \\
\mathcal{E}_{2}(t) & =\frac{M}{2}\left|z_{t}(t)\right|^{2} \\
& =\frac{1}{2} M\left|\frac{d}{d t}\left(F\left(t+l_{1}\right)-G(t)\right)\right|^{2}, \\
\mathcal{E}_{3}(t) & =\frac{\mu_{2}}{2} \int_{0}^{l_{2}}\left|v_{t}(x, t)\right|^{2}+\left|v_{x}(x, t)\right|^{2} d x \\
& =\mu_{2} \int_{0}^{l_{2}}\left|E^{\prime}(t+x)\right|^{2}+\left|H^{\prime}(t+x)\right|^{2} d x .
\end{array}\right\}
$$

Thus $\mathcal{E}_{1}$ is the energy of the part of the string to the left of the point mass, $\mathcal{E}_{2}$ is the energy of the point mass, and $\mathcal{E}_{3}$ is the energy of the part of the string to the right of the mass. We know that the total energies of finite energy solutions of the system do not decay uniformly with respect to the energy norm, but the following theorem shows that $\mathcal{E}_{2}(t)$ and $\mathcal{E}_{3}(t)$ do decay uniformly as $t \rightarrow \infty$. This is obviously because the dissipative boundary condition directly affects the string to the right of the mass and this is apparently enough to deprive the mass of energy as well.

Theorem 1 There exists a constant $C$ such that all finite energy solutions of the string-mass system satisfy, for $t>0$ :

$$
\begin{aligned}
\mathcal{E}_{3}(t) & \leq C \mathcal{E}(0) / t \\
\mathcal{E}_{2}(t) & \leq C \mathcal{E}(0) / \sqrt{t} .
\end{aligned}
$$

Proof of Theorem 1. We break the proof into three Parts. In Parts 1 and 2 we establish (21) and in Part 3 we establish (22). We may assume throughout the proof that the initial velocity of the point mass vanishes because, by Lemma 3 this may be achieved by subtracting a solution of the system that is constructed from an eigenfunction. Hence we assume without loss of generality that the term $F^{\prime}\left(l_{1}\right)-G^{\prime}(0)$ that appears in Equations (14), (15), (16) and (17) is zero.

(Part 1). We start by analyzing $H^{\prime}(t)$ which, for $\sigma>0$, is $e^{\sigma t}$ times the inverse Fourier transform of $h(\sigma+i \xi)$. Specifically,

$$
e^{-\sigma t} H^{\prime}(t)=\lim _{R \rightarrow \infty} \frac{1}{2 \pi} \int_{-R}^{R} h(\sigma+i \xi) e^{i \xi t} d \xi
$$

which converges in $L^{2}(\mathbb{R})$ because $\xi \rightarrow h(\sigma+i \xi)$ is in $L^{2}(\mathbb{R})$. We rewrite $H^{\prime}(t)$ as a sum of "good" and "bad" parts, $H^{\prime}(t)=H_{g}^{1}(t)+H_{b}^{1}(t)$, where

$$
e^{-\sigma t} H_{g}^{\prime}(t)=\lim _{R \rightarrow \infty} \frac{1}{2 \pi} \int_{-R}^{R} h_{g}(\sigma+i \xi) e^{i \xi t} d \xi
$$




$$
e^{-\sigma t} H_{b}^{\prime}(t)=\lim _{R \rightarrow \infty} \frac{1}{2 \pi} \int_{-R}^{R} h_{b}(\sigma+i \xi) e^{i \xi t} d \xi
$$

and

$$
\left.\begin{array}{c}
h_{g}(\lambda)=\frac{h^{1}(\lambda)+q e^{-l_{2} \lambda} e^{1}(\lambda)}{1-q e^{-2 l_{2} \lambda}}, \\
h_{b}(\lambda)=S(\lambda) w(\lambda), \\
w(\lambda)=2 q \mu_{1} e^{-2 l_{2} \lambda}\left(e^{-l_{1} \lambda} f^{1}(\lambda)+g^{1}(\lambda)\right) \\
-2 q \mu_{2}\left(1-e^{-2 l_{1} \lambda}\right)\left(1-q e^{-2 l_{2} \lambda}\right)^{-1}\left(h^{1}(\lambda)+q e^{-l_{2} \lambda} e^{1}(\lambda)\right) .
\end{array}\right\}
$$

We analyze the "good" functions in this part of the proof, and we leave the "bad" functions to Part 2 of the proof. The reason for writing the functions this way is that the decay properties of $H_{g}^{\prime}$ are easily seen and the convergence properties of the integral defining $H_{b}^{\prime}$ are better than those of the corresponding integral for $H^{\prime}$.

An explicit formula for $H_{g}^{\prime}(t)$ exists. In order to write this, we first define:

$$
\begin{aligned}
& H_{c}^{\prime}(t)= \begin{cases}H^{\prime}(t), & t \in\left[0, l_{2}\right], \\
0, & t \in(-\infty, 0) \cup\left(l_{2}, \infty\right),\end{cases} \\
& E_{c}^{\prime}(t)= \begin{cases}E^{\prime}(t), & t \in\left[0, l_{2}\right], \\
0, & t \in(-\infty, 0) \cup\left(l_{2}, \infty\right),\end{cases} \\
& F_{c}^{\prime}(t)= \begin{cases}F^{\prime}(t), & t \in\left[0, l_{1}\right], \\
0, & t \in(-\infty, 0) \cup\left(l_{1}, \infty\right),\end{cases} \\
& G_{c}^{\prime}(t)= \begin{cases}G^{\prime}(t), & t \in\left[0, l_{1}\right], \\
0, & t \in(-\infty, 0) \cup\left(l_{1}, \infty\right),\end{cases}
\end{aligned}
$$

Noting that by (10), $h^{1}$ and $e^{1}$ are the Laplace transforms of $H_{c}^{\prime}$ and $E_{c}^{\prime}$ respectively, we observe that

$$
H_{g}^{\prime}(t)=\sum_{k=0}^{\infty} q^{k}\left[H_{c}^{\prime}\left(t-2 k l_{2}\right)+q E_{c}^{\prime}\left(t-(2 k+1) l_{2}\right)\right] .
$$

Owing to the fact that $H_{c}^{\prime}$ and $E_{c}^{\prime}$ have support in $\left[0, l_{2}\right]$,

$$
\left(\int_{2 m l_{2}}^{2(m+1) l_{2}}\left|H_{g}^{\prime}(t)\right|^{2} d t\right)^{\frac{1}{2}} \leq|q|^{m}\left(\left\|H_{c}^{\prime}\right\|_{L^{2}\left(0, l_{2}\right)}+\left\|E_{c}^{\prime}\right\|_{L^{2}\left(0, l_{2}\right)}\right) .
$$

We note that, because $|q|<1$, the term in the energy corresponding to $H_{g}^{\prime}$ decays exponentially with time. We return to this estimate at the end of Part 2 of the proof. This completes Part 1 of the proof.

Before starting Part 2 of the proof of Theorem 1, we state and prove an inequality that we will need in Part 2.

Lemma 4 If $\nu, \theta, \mu$ and $k$ are real and $k>0,|\nu|<\mu$ then

$$
\left|\frac{(\nu+i \theta)^{k}}{(\mu+i \theta)^{k+1}}\right|^{2} \leq \frac{1}{(k+1)\left(\mu^{2}-\nu^{2}\right)}
$$


Proof. Differentiating the expression with respect to $\theta$ shows that a maximum occurs at $\theta=0$ if $k \mu^{2}-(k+1) \nu^{2} \leq 0$ and at $\theta^{2}=k \mu^{2}-(k+1) \nu^{2}$ if $k \mu^{2}-(k+1) \nu^{2}>0$. In both cases, one easily sees that the inequality is satisfied. this completes the proof of the lemma.

\section{Proof of Theorem 1 (Part 2).}

By (10) and Plancherel's identity, if $\sigma>0$ then the $L^{2}$ norms of $f^{1}(\sigma+i \xi), g^{1}(\sigma+i \xi)$, $h^{1}(\sigma+i \xi)$, and $e^{1}(\sigma+i \xi)$, as functions of $\xi$, are no greater than the $L^{2}$ norms of $F_{c}^{\prime}(t), G_{c}^{\prime}(t)$, $H_{c}^{\prime}(t)$ and $E_{c}^{\prime}(t)$ respectively. This, and the estimate of $S(\lambda)$ in Lemma 1 , show that the integral defining $H_{b}^{\prime}(t)$ converges absolutely. Thus, we may write

$$
H_{b}^{\prime}(t)=\frac{e^{\sigma t}}{2 \pi} \int_{-\infty}^{\infty} h_{b}(\sigma+i \xi) e^{i \xi t} d \xi=\frac{1}{2 \pi i} \int_{\sigma-i \infty}^{\sigma+i \infty} h_{b}(\lambda) e^{\lambda t} d \lambda
$$

We would like to deform the contour defining $H_{b}^{\prime}$ so that it ends up as a line parallel to the imaginary axis in the left half plane. But unfortunately this is impossible because, unlike $h_{g}$, $h_{b}$ has a sequence of poles converging to the imaginary axis. These poles in fact correspond to the sequence of eigenvalues converging to the imaginary axis which has been investigated in [1]. However, there is a way to get around this problem. We define $h_{b, m}(\lambda)=S_{m}(\lambda) w(\lambda)$ (see $(25))$. Next, we note that

$$
S_{m}(\lambda)-S(\lambda)=e^{-2(m+1) \lambda l_{1}}\left[\frac{M \lambda-\mu_{1}+Q(\lambda) \mu_{2}}{M \lambda+\mu_{1}+Q(\lambda) \mu_{2}}\right]^{m+1} S(\lambda)
$$

This shows that if $t<2(m+1) l_{1}$ then

$$
\int_{\sigma-i \infty}^{\sigma+i \infty}\left(h_{b, m}(\lambda)-h_{b}(\lambda)\right) e^{\lambda t} d \lambda \rightarrow 0
$$

as $\sigma \rightarrow \infty$. Since the expression is independent of $\sigma$, it must be zero for $t<2(m+1) l_{1}$. Thus, for $t$ in this range, we may use $h_{b, m}(\lambda)$ instead of $h(\lambda)$ in the formula for $H_{b}^{\prime}(t)$ :

$$
H_{b}^{\prime}(t)=\frac{1}{2 \pi i} \int_{\sigma-i \infty}^{\sigma+i \infty} h_{b, m}(\lambda) e^{\lambda t} d \lambda
$$

We now consider $\gamma$ satisfying $0<\gamma<\delta_{1}=\min \left(\mu_{1} /(2 M),-\log (|q|) /\left(4 l_{2}\right)\right)$ but we shall further restrict $\gamma$ below. We shift the contour in (27) until it becomes the line $\operatorname{Re} \lambda=-\gamma$. Thus we may write

$$
H_{b}^{\prime}(t)=\sum_{k=0}^{m} \zeta_{k}(t) e^{-\gamma\left(t-2 k l_{1}\right)}
$$

where

$$
\zeta_{k}(t)=\frac{1}{2 \pi} \int_{-\infty}^{\infty} w(-\gamma+i \xi) e^{\left(t-2 l_{1}\right) i \xi} \frac{\left[M(-\gamma+i \xi)-\mu_{1}+Q(-\gamma+i \xi) \mu_{2}\right]^{k}}{\left[M(-\gamma+i \xi)+\mu_{1}+Q(-\gamma+i \xi) \mu_{2}\right]^{k+1}} d \xi .
$$

By Lemma 1 , since $\gamma<-\log (|q|) /\left(4 l_{2}\right)$, we must have $\operatorname{Re} Q(-\gamma+i \xi)>\rho=\frac{1-|q|^{1 / 2}}{1+|q|^{1 / 2}}$ for all $\xi \in \mathbb{R}$. Applying Lemma 4, we see that if $0<\gamma<\delta_{2}=\min \left(\delta_{1}, \rho \mu_{2} /(2 M)\right)$, then

$$
\begin{aligned}
\frac{\left|M(-\gamma+i \xi)-\mu_{1}+Q(-\gamma+i \xi) \mu_{2}\right|^{k}}{\left|M(-\gamma+i \xi)+\mu_{1}+Q(-\gamma+i \xi) \mu_{2}\right|^{k+1}} & \leq\left[4 \mu_{1}(k+1)\left(\operatorname{Re} Q(-\gamma+i \xi) \mu_{2}-M \gamma\right)\right]^{-\frac{1}{2}} \\
& <\left[2 \rho \mu_{1} \mu_{2}(k+1)\right]^{-\frac{1}{2}}
\end{aligned}
$$


Hence, by Plancherel's identity, there is a constant $B$ such that

$$
\left\|\zeta_{k}\right\|_{L^{2}(\mathbb{R})} \leq C(\mathcal{E}(0))^{\frac{1}{2}}(k+1)^{-\frac{1}{2}}
$$

Thus

$$
\left(\int_{2 m l_{1}}^{2(m+1) l_{1}}\left|H_{b}^{\prime}(t)\right|^{2}\right)^{\frac{1}{2}} \leq B(\mathcal{E}(0))^{\frac{1}{2}} \sum_{k=0}^{m} \frac{e^{-2 \gamma(m-k) l_{1}}}{\sqrt{k+1}} .
$$

In order to estimate the sum on the right side of this inequality, we use the fact that $e^{-\gamma l_{1}(m-k)} / \sqrt{k+1}$, as a function of $k$, is concave up. Thus, if its value for $k=0$ is no greater than its value for $k=m$ then its value for all $0 \leq k \leq m$ is no greater than its value for $k=m$. That is, if $m$ is large enough to make

$$
e^{-\gamma l_{1} m} \leq \frac{1}{\sqrt{m+1}}
$$

then $e^{-\gamma l_{1}(m-k)} / \sqrt{k+1} \leq 1 / \sqrt{m+1}$ and thus

$$
\frac{1}{\sqrt{k+1}} \leq \frac{e^{\gamma l_{1}(m-k)}}{\sqrt{m+1}}
$$

Consequently

$$
\left(\int_{2 m l_{1}}^{2(m+1) l_{1}}\left|H_{b}^{\prime}(t)\right|^{2}\right)^{\frac{1}{2}} \leq B(\mathcal{E}(0))^{\frac{1}{2}} \sum_{k=0}^{m} \frac{e^{-\gamma(m-k) l_{1}}}{\sqrt{m+1}}=B(\mathcal{E}(0))^{\frac{1}{2}} \frac{1}{\left(1-e^{-\gamma l_{1}}\right) \sqrt{m+1}} .
$$

Thus, by (26) and (28),

$$
\int_{0}^{l_{2}}\left|H^{\prime}(t+x)\right|^{2} d x \leq \frac{\text { constant }}{t} \mathcal{E}(0) .
$$

The analysis for $E^{\prime}$ is similar. This completes Part 2 of the proof and establishes (21).

Proof of Theorem 1 (Part 3). Here we establish (22). It is easy to see that the Laplace transform of $z^{\prime}(t)=F^{\prime}\left(t+l_{1}\right)-G^{\prime}(t)$ is given by

$$
j(\lambda)=e^{\lambda l_{1}}\left(f(\lambda)-f_{1}(\lambda)\right)-g(\lambda) .
$$

Equations (14) and (15) may be used to show that (keeping in mind that we may assume that $\left.F^{\prime}\left(l_{1}\right)-G^{\prime}(0)=0\right)$

$$
\begin{aligned}
j(\lambda) & =\left\{2 \mu_{1}\left(q e^{-2 \lambda l_{2}}-1\right)\left(f_{1}(\lambda) e^{-\lambda l_{1}}+g_{1}(\lambda)\right)\right. \\
& \left.+\left(1-e^{-2 \lambda l_{1}}\right)\left(2 \mu_{2}\left(h_{1}(\lambda)+q e^{-\lambda l_{2}} e_{1}(\lambda)\right)\right)\right\} S(\lambda) .
\end{aligned}
$$

The form of this expression allows a treatment that is identical to that for $h_{b}(\lambda)$ in Part 2 . Thus $z^{\prime}(t)$ satisfies an estimate analogous to (42), i.e.

$$
\int_{0}^{l_{2}}\left|z^{\prime}(t+x)\right|^{2} d x \leq \frac{\text { constant }}{t} \mathcal{E}(0)
$$


But

$$
\begin{aligned}
l_{2} z^{\prime}(t)^{2} & =\int_{0}^{l_{2}} \frac{d}{d x}\left[\left(x-l_{2}\right)\left(z^{\prime}(t+x)^{2}\right] d x\right. \\
& =\int_{0}^{l_{2}} z^{\prime}(t+x)^{2} d x+2 \int_{0}^{l_{2}}\left(x-l_{2}\right) z^{\prime}(t+x) z^{\prime \prime}(t+x) d x \\
& \leq \int_{0}^{l_{2}} z^{\prime}(t+x)^{2} d x+2 l_{2}\left(\int_{0}^{l_{2}} z^{\prime}(t+x)^{2} d x\right)^{\frac{1}{2}}\left(\int_{0}^{l_{2}} z^{\prime \prime}(t+x)^{2} d x\right)^{\frac{1}{2}} .
\end{aligned}
$$

Also, by the last equation of (9),

$$
M z^{\prime \prime}(s)=\mu_{2}\left(H^{\prime}(s)+E^{\prime}\left(s+l_{2}\right)\right)-\mu_{1}\left(F^{\prime}\left(s+l_{1}\right)+G^{\prime}(s)\right),
$$

which yields the estimate

$$
\int_{0}^{l_{2}} z^{\prime \prime}(t+x)^{2} d x \leq \text { constant } \cdot \mathcal{E}(0)
$$

Hence we see from (31), (32) and (43) that

$$
z^{\prime}(t)^{2} \leq \frac{\text { constant }}{\sqrt{t}} \mathcal{E}(0)
$$

which completes the proof of $(22)$.

We now consider what happens if the initial data has an extra order of smoothness between the endpoint $x=-l_{1}$ and the point mass at $x=0$. In this situation, Hansen and Zuazua [1] prove that the corresponding open-loop system is exactly controllable in a finite time interval. We show here that the total energy in our closed loop system decays like a constant divided by time. We make this precise by defining a subspace $\mathcal{Y}$ which contains $\left(U^{0}, V^{0}, Z^{0}, U^{1}, V^{1}, Z^{1}\right) \in \mathcal{H}$ such that

$$
U^{0} \in H^{2}\left(\Omega_{1}\right), U^{1} \in H^{1}\left(\Omega_{1}\right), U^{1}\left(-l_{1}\right)=0, U^{1}(0)=Z^{1}
$$

and we equip $\mathcal{Y}$ with a norm given by

$$
\left\|\left(U^{0}, V^{0}, Z^{0}, U^{1}, V^{1}, Z^{1}\right)\right\|_{\mathcal{Y}}=\left\|\left(U^{0}, V^{0}, Z^{0}, U^{1}, V^{1}, Z^{1}\right)\right\|+\left(\int_{-l_{1}}^{0}\left|U_{x x}^{0}(x)\right|^{2}+\left|U_{x x}^{1}(x)\right|^{2}\right)^{\frac{1}{2}} .
$$

It is easy to check that $\left(\mathcal{Y},\|\|_{\mathcal{Y}}\right)$ is a Banach space. The space $\mathcal{Y}$ is also an invariant subspace of the semigroup $T(t)$. In fact, more is true:

Theorem $2 T(t) \mathcal{Y} \subset \mathcal{Y}$ and the restriction of $T(t)$ to $\mathcal{Y}$ is a strongly continuous semigroup on $\mathcal{Y}$. 
Remark: Because of this, $\mathcal{Y}$ is said to be an $\mathcal{A}$-admissible subspace of $\mathcal{H}$, where $\mathcal{A}$ is the infinitesimal generator of $T(t)$. See Pazy [6] for a discussion of this concept.

Proof. Hansen and Zuazua's proof of Proposition 2.5 in [1] is also applicable here and shows that $T(t) \mathcal{Y} \subset \mathcal{Y}$ and that $T(t)$ is strongly continuous on $\mathcal{Y}$. The semigroup property is obvious because it holds on $\mathcal{H}$.

If $y \in \mathcal{Y}$ then we put $\mathcal{J}(t)=\|T(t) y\|_{\mathcal{Y}}^{2}$. Obviously $\mathcal{J}(t)$ depends on $y$ but the notation does not indicate this dependence.

Theorem 3 Suppose that $y \in \mathcal{Y}$ and $\mathcal{J}(t)=\|T(t) y\|_{\mathcal{Y}}^{2}$. Let $\mathcal{E}$ be the energy associated with the initial data $y$. Then there exists a constant $C$ independent of $y$ such that

$$
\mathcal{E}(t) \leq C \mathcal{J}(0) / t
$$

Proof. By Theorem 1, it is sufficient to show that

$$
\begin{aligned}
& \mathcal{E}_{1}(t) \leq C \mathcal{J}(0) / t \\
& \mathcal{E}_{2}(t) \leq C \mathcal{J}(0) / t
\end{aligned}
$$

The method of proof is similar to that of Theorem 1. We start with

$$
e^{-\sigma t} F^{\prime}(t)=\lim _{R \rightarrow \infty} \frac{1}{2 \pi} \int_{-R}^{R} f(\sigma+i \xi) e^{i \xi t} d \xi
$$

which, like (23), converges in $L^{2}(\mathbb{R})$ if $\sigma>0$ (recall that $F^{\prime}(t)$ is defined to be zero for $t<0$ ). But

$$
\frac{e^{\sigma t}}{2 \pi} \int_{-R}^{R} f(\sigma+i \xi) e^{i \xi t} d \xi=\frac{1}{2 \pi i} \int_{\Gamma(\sigma, R)} f(\lambda) e^{\lambda t} d \lambda
$$

where $\Gamma(\sigma, R)$ is any contour in the right half plane that starts at $\sigma-i R$ and ends at $\sigma+i R$. The region of analyticity of $f$ is the same as that of $S$. We see then that $f$ is analytic in a neighborhood of the origin because $S(0)=2 \mu_{1}(1-q)^{-1}$ and $S$ is meromorphic. Because of this, we may allow $\Gamma(\sigma, R)$ to pass to the left of the origin and for our purposes it will be sufficient to choose $\epsilon>0$ sufficiently small and set $\Gamma(\sigma, R)$ to be the union of

- the portion of the circle that is contained in the left half plane and is centered at the origin with radius $\epsilon$,

- the line segments $(\sigma-i R, \sigma-i \epsilon),(\sigma-i \epsilon,-i \epsilon),(i \epsilon, \sigma+i \epsilon),(\sigma+i \epsilon, \sigma+i R)$.

Hence we may now write

$$
F^{\prime}(t)=\lim _{R \rightarrow \infty} \frac{1}{2 \pi i} \int_{\Gamma(\sigma, R)} f(\lambda) e^{\lambda t} d \lambda
$$

and the limit converges in $L_{l o c}^{2}(\mathbb{R})$. 
Now we make use of the extra smoothness of the initial data in the interval $\Omega_{1}$ and integrate the formulae for $f^{1}(\lambda)$ and $g^{1}(\lambda)$ by parts to obtain

$$
\left.\begin{array}{l}
f^{1}(\lambda)=\left(f^{2}(\lambda)+F^{\prime}(0)-F^{\prime}\left(l_{1}\right) e^{-l_{1} \lambda}\right) / \lambda, \\
g^{1}(\lambda)=\left(g^{2}(\lambda)+G^{\prime}(0)-G^{\prime}\left(l_{1}\right) e^{-l_{1} \lambda}\right) / \lambda,
\end{array}\right\}
$$

where

$$
f^{2}(\lambda)=\int_{0}^{l_{1}} e^{-\lambda t} F^{\prime \prime}(t) d t, \quad g^{2}(\lambda)=\int_{0}^{l_{1}} e^{-\lambda t} G^{\prime \prime}(t) d t
$$

As in the proof of Theorem 1, we may assume without loss of generality that $F^{\prime}\left(l_{1}\right)=G^{\prime}(0)$. Further, the Dirichlet boundary condition at $x=-l_{1}$ implies that $G^{\prime}\left(l_{1}\right)=F^{\prime}(0)$. These equations and (39) may be used to rearrange (14), yielding

$$
f(\lambda)=\frac{F^{\prime}(0)}{\lambda}+f_{b}(\lambda)
$$

where

$$
\begin{aligned}
f_{b}(\lambda) & =\frac{S(\lambda)}{\lambda}\left\{f^{2}(\lambda)\left[M \lambda+\mu_{1}+\mu_{2}-q e^{-2 l_{2} \lambda}\left(M \lambda+\mu_{1}-\mu_{2}\right)\right]\right. \\
& +g^{2}(\lambda) e^{-l_{1} \lambda}\left[M \lambda-\mu_{1}+\mu_{2}-q e^{-2 l_{2} \lambda}\left(M \lambda-\mu_{1}-\mu_{2}\right)\right] \\
& \left.+2 \mu_{2} \lambda e^{-l_{1} \lambda}\left(h^{1}(\lambda)+q e^{1}(\lambda) e^{-l_{2} \lambda}\right)+2 \mu_{1} q e^{-\left(l_{1}+2 l_{2}\right) \lambda} G^{\prime}(0)\right\} .
\end{aligned}
$$

Using the fact that

$$
\lim _{R \rightarrow \infty} \int_{\Gamma(\sigma, R)} \frac{e^{\lambda t}}{\lambda} d \lambda=0
$$

we see that

$$
F^{\prime}(t)=\lim _{R \rightarrow \infty} \frac{1}{2 \pi i} \int_{\Gamma(\sigma, R)} f_{b}(\lambda) e^{\lambda t} d \lambda=\frac{1}{2 \pi i} \int_{\Gamma(\sigma, \infty)} f_{b}(\lambda) e^{\lambda t} d \lambda
$$

and the last integral converges absolutely. The remainder of the analysis of $F^{\prime}(t)$ is almost identical to that of $H_{b}^{\prime}(t)$ in Theorem 1, so we omit the details and conclude that

$$
\int_{-l_{1}}^{0}\left|F^{\prime}(t-x)\right|^{2} d x \leq \frac{\text { constant }}{t} \mathcal{J}(0) .
$$

The analysis for $G^{\prime}$ is similar. This establishes (36).

Because of (36), it is easy to see that Estimate (43) may be improved to give

$$
\int_{0}^{l_{2}} z^{\prime \prime}(t+x)^{2} d x \leq \text { constant } \cdot \mathcal{J}(0) / t
$$

which in turn gives an improvement of Estimate (34):

$$
z^{\prime}(t)^{2} \leq \operatorname{constant} \cdot \mathcal{J}(0) / t
$$

This establishes (37) and completes the proof of the theorem. 


\section{References}

[1] S. Hansen and E. Zuazua, Exact Controllability and Stabilization of a Vibrating String with an Interior Point Mass, SIAM J. Control Optim 33 no. 5 (1995) 1357-1391.

[2] F. Huang, Characteristic Conditions for Exponential Stability of Linear Dynamical Systems in Hilbert Spaces, Ann. of Diff. Eq. 1 (1) 1985.

[3] V. Komornik, Controllability and Stabilization: The Multiplier Method, Chichester, New York: Wiley, Paris: Masson (1994).

[4] J. E. Lagnese, G. Leugering and E. J. P. G. Schmidt, Control of Planar Networks of Timoshenko Beams, SIAM J. Control and Optimization 31, No. 3 (May, 1993) 780-811.

[5] W. Littman, L. Markus; Some recent results on control and stabilization of flexible structures, in "Stabilization of Flexible Structures", A. V. Balakrishnan and J. P. Zolesio, eds., Optimization Software Inc., Los Angeles, 1988.

[6] A. Pazy, Semigroups of Linear Operators and Applications to Partial Differential Equations, Springer-Verlag (1983). 